\title{
Redes transnacionales y etnicidad: análisis sociometrico de las redes personales de latinoamericanos y africanos en Barcelona, España
}

\section{Transnational Networks and Ethnicity: sociometric analysis of personal networks of Latin America and Africa in Barcelona, Spain}

\author{
Javier Ávila Molero
}

Universidad Autónoma de Barcelona, Bellaterra, Cerdanyola del Valles, Barcelona, España Egolab - Laboratorio de Redes Personales y Comunidades

\section{Resumen}

Las redes de los inmigrantes son cada vez más complejas. Hoy día se han vuelto multiétnicas y transnacionales. Estas redes constituyen la estructura sobre la cual los inmigrantes desarrollan sus procesos de sociabilidad e identificación étnica. Esta nueva complejidad exige el desarrollo de nuevas metodologías y conceptos para su análisis, que vaya más allá de los datos cualitativos y las metáforas, hacia datos más objetivos y sociometricos. ¿Cómo estudiar las redes de los inmigrantes en esta nueva complejidad? ¿Cómo identificar sus procesos y dinámicas de cambio? En el presente artículo proponemos usar las redes como instrumento de análisis sociometrico, más allá de los usos hermenéuticos predominantes, y desarrollar nuevas variables para el estudio de la "etnicidad práctica" que desarrollan los inmigrantes, en el espacio transnacional. Los resultados del estudio se basan en dos proyectos de investigación desarrollados con colectivos inmigrantes de latinoamericanos y africanos en Barcelona, España, por parte del Laboratorio de Redes Personales y Comunidades del departamento de Antropología de la Universidad Autónoma de Barcelona - UAB.

Descriptores: etnicidad, transnacionalismo, redes, inmigración.

\begin{abstract}
Immigrant networks are increasingly complex. Today they have become multiethnic and transnational. These networks provide the framework on which immigrants develop their socialization processes and ethnic identification. This new complexity requires the development of new methodologies and concepts for analysis beyond qualitative data and metaphors, to more objective and sociometric data. How to study networks of immigrants in this new complexity? How to identify the processes and dynamics of change? In this paper we propose to use the networks as sociometric analysis tool beyond prevailing hermeneutic uses, and develop new variables for the study of "ethnicity practice" developed by immigrants in transnational space. The survey results are based on two research projects developed with groups of American and African immigrants in Barcelona, Spain, by the Laboratory of Personal Networks and Communities Department of Anthropology at the Autonomous University of Barcelona - UAB.
\end{abstract}

Keywords: ethnicity, transnationalism, networks, immigration

\section{Introducción}

El presente artículo propone una nueva metodología para el análisis de la etnicidad de los inmigrantes, a través del estudio de la estructura y composición de sus redes personales, tanto locales, como no-locales, dentro del nuevo escenario transnacional y globalizado.

Para ello utiliza los nuevos avances en el análisis de redes, a través del uso de software especializado como Egonet, que permite el análisis sociometrico de las redes que los inmigrantes desarrollan en su sociabiliad cotidiana; más allá de las auto-percepciones de los propios inmigrantes y los estereotipos de los personas originarias de las sociedades receptoras.

Este giro metodológico nos ofrece una perspectiva más objetiva sobre la etnicidad inmigrante y un enfoque más realista sobre los procesos de pertenencia múltiple, que a nivel social y cultural 
desarrollan los inmigrantes entre las sociedades de destino y origen.

En el análisis proponemos dos nuevos indicadores sociometricos para el estudio de la etnicidad migrante: a) micro-estructura de la red y b) composición étnica de la red. Ambas variables permiten una nueva mirada sobre las propiedades de estructura y composición étnica de las redes de los inmigrantes, locales y no-locales y permiten construir un modelo explicativo sobre sus procesos de cambio en el nuevo escenario transnacional.

Estas reflexiones se fundamentan en los resultados empíricos obtenidos a partir del trabajo de campo desarrollado por el equipo del Laboratorio de Redes Personales y Comunidades (Egolab) de la Universidad Autónoma de Barcelona, a través de dos proyectos de investigación titulados Development of a social network of acculturarion and

its applications to inmigrant populations in south Florida and the northeastern Spain ${ }^{1}$ (2004 - 2006) y Dinamics of actors and networks across levels: individuals, groups, organizations and social settings $^{2}(2006-2008)$.

\section{Redes y transnacionalismo}

Las redes se han convertido en una importante herramienta para el análisis de la migración internacional. De manera frecuente los analistas recurren a conceptos como "cadenas migratorias" (Arango, 2003) [1], "redes migratorias" (Massey et. Al. 2000) [2] o "redes transplantadas" (Tilly, 1990) [3], entre otros, para explicar el desarrollo de los flujos migratorios.

Sin embargo, ha sido entre los analistas de la teoría transnacional donde el concepto de redes ha tenido más éxito.

En efecto, desde la teoría transnacional se recurre frecuentemente a la metáfora de las "redes y conexiones" para explicar las nuevas prácticas de sociabilidad transnacional que los inmigrantes desarrollan entre las sociedades de origen y destino de sus flujos migratorios (Glick Schiller,

\footnotetext{
${ }^{1}$ National Science Foundation, award no BCS-0417429, dirigido por Christopher McCarty en los Estados Unidos y José Luis Molina en España.

2 European Collaborative Research Project (ECRP) financiado por la European Science Foundation y el Ministerio de Ciencia y Tecnología (SEJ2005-25683-E, SEJ2007-29468-E, 05_ECRP_FP026).
}

1992) [4]. En un mundo crecientemente globalizado, definido como "sociedad-red", no extraña que el concepto de redes se haya posicionado como "evidente" para el análisis de las migraciones internacionales.

Sin embargo, el uso común del concepto de redes no estaría exento de problemas. En lo fundamental, en el concepto de redes se utiliza más como metáfora y no como herramienta de análisis. Como metáfora, el concepto de redes se suele entender como los vínculos que desarrollan entre si los inmigrantes de un mismo país de origen. Este sentido conlleva a pensar en vínculos cuya naturaleza social y cultural sería endogámica.

Desde esta perspectiva, cuando se habla de "redes étnicas" se suelo pensar en las redes que los inmigrantes desarrollan con sus connacionales; entendiéndose que en contextos migratorios el origen nacional de los inmigrantes se constituye en uno de los principales registros desde el cual se (re)construye la identificación/diferenciación entre el "nosotros" y los "otros" (Vertovec, 2007) [5].

Desde nuestra perspectiva, la metáfora de redes no permite calibrar, en su real dimensión, el impacto que el cambio de sociedad genera en la redefinición de las redes de los inmigrantes. El proceso migratorio genera un enorme cambio tanto en la estructura y composición de las redes originarias del inmigrante. En la mayoría de casos las redes desarrolladas en la sociedad de origen suelen desaparecer, y las que se mantienen se tienen que "reinventar" en forma y contenido desde el nuevo escenario social transnacional. Esto sucede inclusive con aquellas redes en apariencia menos proclives al cambio, como las de parentesco.

En la sociedad de destino el inmigrante desarrolla nuevas redes, las cuales pueden tener una composición variable de: a) connacionales, b) nativos de la sociedad de destino e c) inmigrantes de otras nacionalidades. La heterogeneidad de estas redes en la sociedad de destino se combina con la complejidad de las redes transnacionales que los inmigrantes desarrollan y mantienen con sus sociedades de origen. 
La complejidad de estas redes desborda el uso tradicional del concepto como metáfora.

Es necesario repensar el concepto de redes en los estudios migratorios, y utilizarlo más como categoría de análisis y no sólo como metáfora de "redes étnicas". Esto parte por comprender que el proceso de reconstrucción de redes por parte de los inmigrantes es duro y complejo; y que no se trata de un simple "transplante" de "redes étnicas" originarias, como muchas veces se sostiene; sino más bien de un nuevo proceso de reinvención de redes, que implica -necesariamente- el desarrollo de una nueva sociabilidad y una nueva cultura. Este proceso de reinvención del conjunto de redes del inmigrante significa también su reinvención como individuo, sus identificaciones y sentidos de comunidad.

De manera tradicional el concepto de "comunidades transnacionales" se ha vinculado con la metáfora de las "redes étnicas". (Rouse, 1991) [6], (castles, 2007) [7], (Guarnizo, 2007) [8]. ¿El problema? Este uso del concepto "comunidad transnacional" presupone, a priori, que las redes de los inmigrantes son socialmente endógenas y culturalmente homogéneas; dejándose fuera del análisis las redes que los mismos inmigrantes también desarrollan con personas de otras nacionalidades, como las personas originarias de la sociedad de destino (España) y/o con inmigrantes de otras países.

Un análisis sociometrico más detallado nos muestra que las redes de los inmigrantes desarrollan una estructura y composición más compleja y que a su interior se desarrolla una tensión, fuerte, entre redes endógenas y homogéneas, de un lado, y redes exógenas y heterogéneas, de otro lado.

De esta manera, si el concepto tradicional de "comunidad transnacional" sólo se enfoca en los vínculos endógenos y homogéneos se ofrece una versión parcial de complejidad de las comunidades transnacionales, invisibilizando procesos reales de pertenencias múltiples, entre diferentes comunidades.

Nuestra propuesta es repensar el concepto de redes étnicas desde su análisis sociometrico y estudiar desde la perspectiva sugerida por Alejandro Portes (2003) [9] "al individuo y sus redes sociales", como unidad de análisis en los estudios transnacionales.

Esta nueva perspectiva podría permitir repensar los procesos de identificación étnica de los inmigrantes desde las características de sus redes, entendiendo que si la cultura es una red de símbolos (Geertz, 1982) [10], hay que entender que estos se realizan precisamente en el plano de la comunicación e interacción entre personas. Es decir, las redes.

En la actualidad es posible dar un giro de tuerca al uso tradicional del concepto de redes como metáfora y usarlo como herramienta de análisis. Esto es posible gracias a los desarrollos teóricos en el campo de las redes personales (Wellman, 1988; 1996) [11], (Molina, 2007) [12] y las nuevas metodologías de investigación que incluyen el uso de nuevos softwares especializados, como Ucinet y Egonet, que permiten estudiar con altos niveles de precisión el conjunto de los vínculos redes personales que los inmigrantes desarrollan entre sus sociedades de destino y origen, pero también con personas originarias de la sociedad receptora.

\section{Redes étnicas: de la metáfora al análisis sociometrico}

Por lo general, las comunidades transnacionales han sido entendidas como entidades cerradas y homogéneas, de fuertes vínculos internos, constituidas por inmigrantes de una misma nacionalidad de origen y residencia geográfica cercana en la misma sociedad de destino. Esta sería una forma incompleta de entenderlas, porque no incluye, en la definición, al conjunto de redes realmente existentes que sus miembros desarrollan, las que incluyen también a nativos de la sociedad receptora e inmigrantes de otras naciones de origen.

Hoy en día, con la revolución de las comunicaciones y el incremento de la movilidad geográfica, las redes personales de los inmigrantes son cada vez más complejas y su perfil sociocultural más diverso.

Esta complejidad se puede apreciar en la Fig. 1, que representan las redes de un inmigrante varón en Barcelona (España). Los nodos del círculo interno (negro) representa los contactos del inmigrante (centro del cuadrante) en España y los nodos del segundo circulo (rojo) representa los contactos del inmigrante en su país de origen. La forma de los nodos representa la nacionalidad de 
origen de cada contacto, donde el circulo representa Españoles, los triángulos inmigrantes de otros países y los cuadrados connacionales.

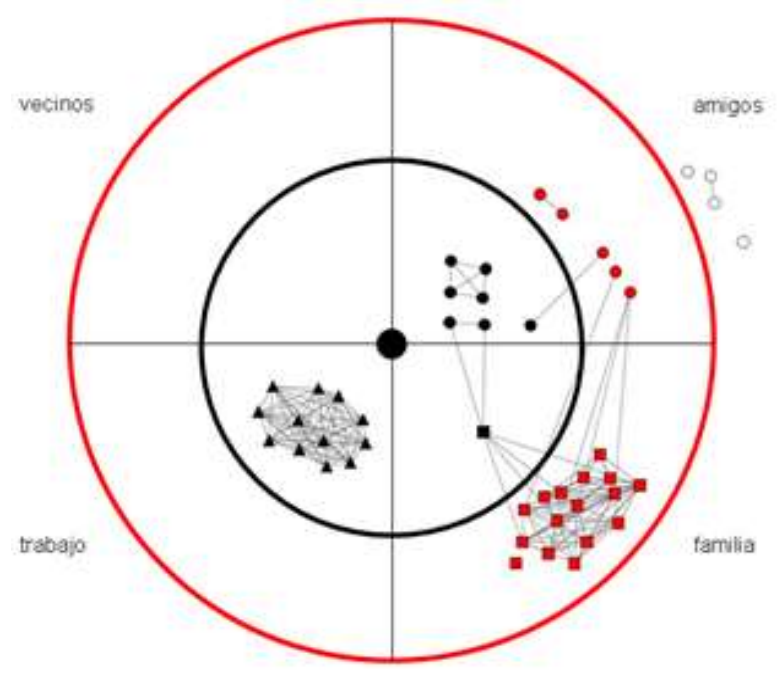

Fig. 1: Representación de las redes personales

La Fig. 1 representa de manera sencilla y elemental el nuevo tipo de redes que los inmigrantes desarrollan en la actualidad: multiétnicas y transnacionales. Se trata de las nuevas "redes étnicas" que los inmigrantes desarrollan en un mundo más globalizado, transnacional y multicultural, diferentes a las que el sentido común convencional, propio de las metáforas de las redes étnicas, suele sostener.

\section{Metodología de la investigación}

La investigación se desarrolló con cuatro colectivos inmigrantes de América Latina y África en Barcelona: Argentina, República Dominicana, Marruecos y Senegal. Las poblaciones analizadas se escogieron tomando en consideración la antigüedad de sus procesos migratorios así como las diferencias étnicas existentes entre cada uno de las poblaciones, según criterios lingüísticos, religiosos y "raciales". Nuestro objetivo era utilizar el análisis de redes como herramienta para el estudio transcultural de los inmigrantes, no tanto desde el ámbito narrativo de sus representaciones sobre la integración y el transnacionalismo, sino desde las prácticas de sociabilidad realmente existentes que desarrollan en sus vidas cotidianas.

En su primera fase, el proyecto recogió 300 redes personales (de manera aleatoria) a inmigrantes con tiempo de residencia en España de entre 2 a 10 años. En el trabajo de campo se utilizó el software especializado en análisis de redes personales denominado Egonet.

Egonet permite el diseño de una encuesta para que la persona entrevistada identifique un número determinado de personas con las que mantiene vínculos. En nuestro caso, definimos recoger una muestra de 45 personas para reconstruir la red de cada entrevistado. La encuesta consta de 4 módulos: a) sobre las características del entrevistado, b) sobre las 45 personas con las que tiene vínculos, c) las características de estas 45 personas y d) los vínculos entre estas 45 personas. Al final, Egonet permite visualizar la red personal con la información recogida en la encuesta.

La segunda fase del proyecto se desarrolló dos años después de la primera. En esta segunda fase se escogió una muestra de 80 casos entre los 300 originalmente estudiados, a los que se volvió a aplicar una segunda encuesta de redes personales con Egonet, manteniendo los mismos criterios metodológicos de la primera fase del estudio.

\section{Redes Personales}

Existe la creencia generalizada de que las personas desarrollamos nuestros vínculos de sociabilidad de manera aleatoria. Sin embargo, más allá del libre albedrío del individuo, lo cierto es que estos vínculos asumen cierta estabilidad en el tiempo, convirtiéndose en redes sociales. En su conjunto, estas redes van constituyendo "microestructuras" de sociabilidad, a través de las cuales interactúan las personas con sus contactos. En el caso de los inmigrantes transnacionales, el estudio del conjunto de sus redes sociales personales nos ofrece una radiografía de las "micro-estructuras" que conforman sus ámbitos de sociabilidad transnacional.

En su sentido original, el estudio de las redes personales se enfocaba en el análisis de los "lazos fuertes" (Wellman 1988), los cuales solían estar compuestos, en promedio, por una docena de "alteri" o contactos.' Sin embargo, de manera reciente, Chris McCarty (2002) y José Luis Molina (2007) han recomendado extender el foco del análisis de las redes personales hacia el conjunto de su comunidad personal.

Para esto, se requiere ampliar el número de "alteri" o "contactos" al menos a 45. Esta cantidad ofrecería una muestra más confiable sobre el conjunto de las redes personales de un individuo y permite que el análisis se amplíe desde el núcleo 
restringido de "lazos fuertes" hacia la periferia más extensa de sus "lazos débiles" (Molina 2007).

El estudio de las redes personales con una muestra de 45 alteri o contactos es complejo. Para ello se requiere del auxilio de software especializado en recolección, análisis y visualización de datos reticulares, como Egonet, el cual nos ha permitido identificar dos variables importantes para el análisis de las redes étnicas de los inmigrantes: a) micro-estructura y b) composición étnica. En el primer caso, se refiere a los "cluster" existentes dentro de la red personal del inmigrante. En el segundo caso, se refiere al origen nacional de las personas que forman parte de la comunidad personal del inmigrante.

\section{Micro-estructuras: "cluster" o grupos en las redes personales}

En nuestra investigación hemos identificado tres tipos de cluster: a) concentrado (1 grupo), b) dual (2 grupos) y c) segmentado (tres o más grupos). Las redes de cluster "concentrado" se caracterizan por su entramado de alta densidad interna, agrupados en un único cluster. Las de cluster "duales" desarrollan un entramado de densidad intermedia, agrupados en torno a dos clusters. Finalmente, las redes de cluster "segmentados" desarrollan un entramado de redes sociales de baja densidad, dispersos entre tres o más clusters.

La Fig. 2 representa un ejemplo de cada tipo de micro-estructura:
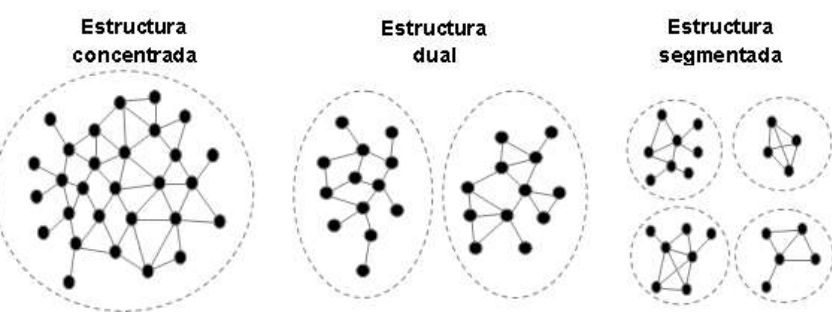

Fig. 2: Micro estructuras de redes personales

En la investigación hemos encontrado que la estructura más común en las redes personales de los inmigrantes es la de tipo "concentrada". Este tipo de estructura se desarrolla en el $47 \%$ de los casos analizados, tal como se puede apreciar en las cifras de la Tabla 1:
Tabla 1: Estructura de cluster en redes personales inmigrantes

\begin{tabular}{|l|c|c|}
\hline Tipo de redes & \% & Cantidad \\
\hline Concentradas & 47 & 132 \\
\hline Duales & 26 & 72 \\
\hline Segmentadas & 27 & 74 \\
\hline Total & 100 & 278 \\
\hline
\end{tabular}

\section{Composición étnica}

La composición étnica de la red personal del inmigrante ofrece información sobre sus procesos de integración en la sociedad receptora.

Por lo general, este proceso se ha estudiado a partir de las narrativas de los inmigrantes. El análisis de las redes personales permite estudiar estos procesos desde sus mismas prácticas de sociabilidad, las cuales se pueden desarrollar con las siguientes opciones: a) personas de la nacionalidad de origen, b) de la sociedad de destino y c) de otras nacionalidades.

Para identificar los niveles de composición étnica se ha diseñado un nuevo indicador que considera la proporción de vínculos del inmigrante con personas de su nacionalidad de origen y españoles.

A mayor número de connacionales (y menor de españoles) la composición étnica de la red será más segregada; mientras que a mayor número de españoles (y menor de connacionales) la composición étnica de la red será más asimilada.

Los rangos son los siguientes:

a) composición segregada (31 a 45 connacionales), b) composición hibrida (16 a 30 connacionales) y

c) composición asimilada (1 a 15 connacionales).

Los siguientes Figs muestran ejemplos de cada caso (los contactos de la misma nacionalidad del inmigrante se representan en color negro, y los de la sociedad de destino en color blanco).

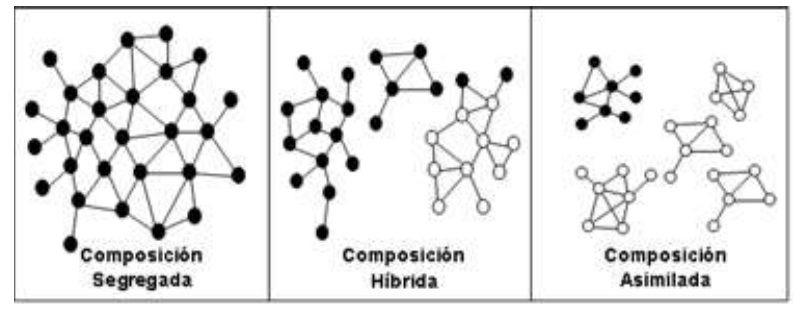

Fig. 3: Composición étnica de las redes 
En la investigación hemos encontrado que el tipo de composición étnica más común es la segregada. Este tipo de composición se desarrolla en el $49 \%$ de los casos, tal como se puede apreciar en la Tabla 2:

Tabla 2: Composición étnica de las redes

\begin{tabular}{|l|c|c|}
\hline Composición & \% & Cantidad \\
\hline Segregada & 49 & 137 \\
\hline Mixta & 28 & 78 \\
\hline Asimilada & 23 & 63 \\
\hline Total & 100 & 278 \\
\hline
\end{tabular}

¿Existe alguna relación entre los cluster de las redes y la composición étnica? En nuestra investigación hemos encontrado una respuesta afirmativa.

Esta relación sería positiva entre los cluster concentrados y la composición étnica segregada $(59 \%$, siendo el promedio $49 \%$ ) y entre la estructura segmentada y la composición étnica asimilada (30\%, siendo el promedio $23 \%$ ). Esto significa que existiría una tendencia a que las redes de estructura concentrada tengan una composición más homogénea y las redes de estructura más segmentada una composición más asimilada.

En Tabla 3 muestra estas cifras con mayor detalle.

Tabla 3: Composición y estructura de red (\%)

\begin{tabular}{|l|c|c|c|c|}
\hline & \multicolumn{3}{|c|}{ Composición } & \\
\hline Estructura & Segregada & Híbrida & Asimilada & Total \\
\hline Concentrada & 59 & 24 & 17 & 100 \\
\hline Dual & 47 & 28 & 25 & 100 \\
\hline Segmentada & 35 & 35 & 30 & 100 \\
\hline Total & 49 & 28 & 23 & 100 \\
\hline
\end{tabular}

¿Qué ocurre con las redes duales? Nuestra hipótesis es que estas redes tendrían una ubicación intermedia entre los extremos de las redes más segregadas y concentradas, por un lado, y las redes más asimiladas y segmentadas, por otro lado.

El siguiente modelo representa esta relación entre micro-estructura y composición étnica en las redes personales de los inmigrantes:

\section{Sociabilidad transnacional.}

Las redes de micro estructura más concentrado y composición étnica más segregada desarrollan redes étnicas transnacionales distintas a las de estructuras más segmentadas y composición más asimilada.

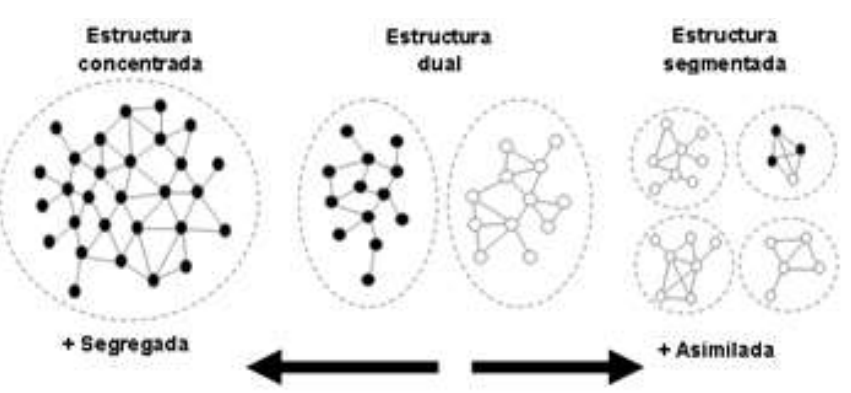

Fig. 4: Estructuras cluster y composición étnica de las redes personales transnacionales

Las redes de composición segregadas suelen desarrollar un campo transnacional más "amplio". En este caso, la condición transnacional suele involucrar varias esferas de la vida social del inmigrante, desarrollando una centralidad importante en su vida cotidiana. Por su parte, las redes de composición asimilada suelen desarrollar un campo transnacional más "reducido", circunscrito a una esfera más específica de la vida social del inmigrante.

Una rápida comparación entre ambos tipos de redes personales nos muestra que las "segregadas" se caracterizan por fuertes vínculos de apoyo social interno, con redes que asumen múltiples funciones. La sociabilidad del individuo tiende a ser más comunitarista, y sus expresiones identitarias se relacionan con la búsqueda de la "reproducción" de la cultura originaria.

Por su parte, las redes más "asimiladas" se caracterizan por tener vínculos más definidos y especializados. La sociabilidad suele ser más individualista y sus expresiones identitarias más relacionadas a la hibridación de la cultura originaria y/o la asimilación de los códigos culturales de la sociedad de origen.

\section{Dinámicas de cambio en las redes personales}

Las redes personales son dinámicas. Presentan cambios en su estructura y composición. En nuestro caso, para identificar las dinámicas del cambio construimos otro indicador que nos permita identificar la variación de contactos la red personal de un inmigrante en dos periodos diferentes de tiempo. 
Tomando en cuenta el número de nuevos contactos en la red del inmigrante podíamos identificar si el nivel del cambio, que podía ser:

a) fuerte (el cambio era entre 31 a 45 contactos),

b) medio (el cambio era entre 16 a 30 contactos y

c) débil (el cambio era entre 1 a 15 contactos).

Al momento de iniciar la segunda fase de la investigación, nuestra hipótesis era que había una relación entre la estructura y composición de las redes personales con sus dinámicas de cambio. De manera específica, sosteníamos que las redes de estructura segmentada y composición asimilada serían, por su naturaleza más "abierta" y "volátil", redes más proclives al cambio; mientras que las redes de estructura concentrada y composición segregada serían, también por su naturaleza "cerrada" y "densa", más proclives a la estabilidad. Sin embargo, los datos recogidos en la segunda fase del estudio falsaron nuestra hipótesis.

En primer lugar, encontramos que el grupo que suponíamos iba a desarrollar menos cambios, el de Senegal -por su estructura concentrada y composición homogénea-, fue el que desarrolló mayores porcentajes de "cambio fuerte" (56\%); mientras que el grupo que suponíamos iba a desarrollar mayores cambios, el argentino -por su estructura segmentada y composición heterogénea-, fue el grupo con menor nivel de cambio "cambio fuerte" (18\%).

Tabla 4: Nivel de cambio en las redes por poblaciones inmigrantes (\%)

\begin{tabular}{|l|c|c|c|c|}
\hline & \multicolumn{3}{|c|}{ Nivel de cambio } & \\
\hline inmigrantes & Fuerte & Medio & Débil & Total \\
\hline Argentina & 11 & 71 & 18 & 100 \\
\hline $\begin{array}{l}\text { República } \\
\text { Dominicana }\end{array}$ & 47 & 47 & 6 & 100 \\
\hline Marruecos & 46 & 54 & 0 & 100 \\
\hline Senegal & 56 & 44 & 0 & 100 \\
\hline Total & 39 & 54 & 7 & 100 \\
\hline
\end{tabular}

Total: 61

En segundo lugar, encontramos que el tipo de estructura más proclive al cambio era la "dual" y no la de tipo "segmentado".

Del $100 \%$ de casos que presentaron relación positiva entre cambio de contacto y cambio de estructura de cluster, el $74 \%$ ocurrió con estructuras que originalmente eran "dual", tal como se aprecia en la Tabla 5:
Tabla 5: Cambio en la estructura de redes (\%)

\begin{tabular}{|l|c|c|c|}
\hline \multirow{2}{*}{ Estructura } & \multicolumn{2}{|c|}{ Cambio } & \\
\cline { 2 - 4 } & Si & No & Total \\
\hline Concentrada & 23 & 77 & 100 \\
\hline Bipolar & 74 & 26 & 100 \\
\hline Segmentada & 38 & 62 & 100 \\
\hline Total & 43 & 57 & 100 \\
\hline
\end{tabular}

Total: 61

Las cifras del Tabla muestran con claridad que la estructura de tipo "dual" es la más proclive al cambio. ¿Por qué motivo? Nuestra hipótesis es que la estructura "dual" no sólo sería una estructura intermedia entre las estructuras "concentrada" y "segmentada", sino también una fase estructural de tránsito entre las dos principales dinámicas del cambio en la estructura de los cluster: a) la "segmentación" y b) la "concentración".

En las dinámicas de cambio en las comunidades personales, el proceso de "concentración" de los cluster suele ir acompañado de otro de "segregación" de su composición étnica. Del mismo modo, el proceso de "segmentación" de los cluster suele ir acompañado por otro de "asimilación" en su composición étnica.

El siguiente modelo representamos esta relación dialéctica entre los procesos de "segmentación" y "concentración" de las estructuras de cluster, con los de "segregación" y "asimilación" de su composición étnica. Se puede apreciar como las redes "duales" tienen una ubicación intermedia, de "transito estructural", entre los extremos de las redes concentradas y segregadas, por un lado, y las redes segmentadas y asimiladas, por otro lado.

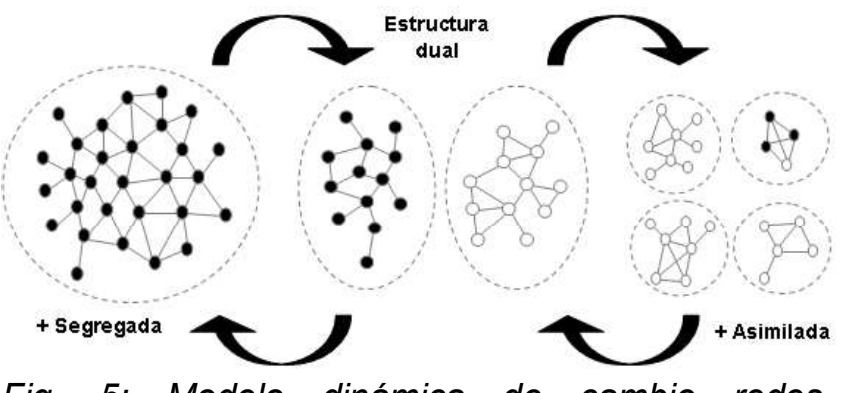

Fig. 5: Modelo dinámica de cambio redes personales inmigrantes

\section{Conclusiones}

La micro-estructura y la composición étnica de las redes personales son dos variables importantes para el estudio de las redes transnacionales. 
En el caso de los cluster hemos identificado tres tipos: a) concentrado, b) dual y c) segmentado.

En el caso de la composición étnica hemos identificado tres tipos diferentes: a) segregadas, b) híbridas y c) asimiladas.

En la investigación hemos encontrado una relación entre la estructura de cluster de las redes y su composición étnica.

Las redes de cluster concentradas tienden a tener una composición étnica más segregada, mientras que en el caso de las estructuras segmentadas, la composición étnica suele ser más asimilada.

Las estructuras duales se ubican en una situación intermedia.

Las redes personales son dinámicas. A nivel de micro-estructura hemos identificado dos grandes procesos de cambio: a) "concentración" y b) "segmentación".

A nivel de composición étnica hemos identificado otros dos procesos: a) segregación y b) asimilación.

Los procesos de concentración en la estructura de cluster suelen ir acompañados de un proceso de segregación en su composición étnica y los procesos de segmentación suelen ir acompañado de otro de asimilación en su composición étnica.

\section{Referencias}

[1] Arango, Joaquín (2003) "La población mundial", en Romero, J. (coord.) Geografía Humana, Barcelona: Ariel, pp. 55-99. España.

[2] Massey, Douglas S. et al (2000) "Teorías sobre la migración internacional: una reseña y una evaluación", en: Revista Trabajo, año 2, Número 3, Enero del 2000.

[3] Tilly, Charles. (1990) 'Transplanted networks,' in Immigration Reconsidered, V. Yans-MacLoughlin (Ed.), New York: Oxford University Press, pp. 7995.

[4] Glick Schiller, Nina, Linda Basch y Cristina Szanton-Blanc (1992) "Transnationalism: A new Analytic Framework for Understanding Migration", en: N. Glick Schiller, L. Basch y C. Blanc-Szanton (Eds.), Towards a Transnational Perspective on Migration: Race, Class, Ethnicity and Nationalism Reconsidered. New York Academy of Sciences, New York.

[5] Vertovec, Steven, (2007) Super-diversity and its implications. Ethnic and Racial Studies, vol 30, núm 6 , pp 1024-1054.
[6] Rouse, Roger (1991) 'Mexican migration and the Social Space of Postmodernism', en: Diaspora, Vol. 1 (Spring), pp. 8-23.

[7] Castles, Stephen (2007) "transnacional Communities: a new form of social relations Ander conditions of grobalization?", en: Reitz J. (ed.) Host Societies and the reception of immigrants. Center for comparative immigrtion studies, University of California, San Diego.

[8] Guarnizo, Luis (2007) "Aspectos económicos del vivir transnacional", en: Ariza M. y Portes A. (eds.) El país transnacional: migración mexicana y cambio social a través de la frontera, Universidad Nacional Autónoma de México, Instituto de Investigaciones Sociales. México.

[9] Portes, Alejandro, Luis Guarnizo y Patricia Landlot (2003) La globalización desde abajo: transnacionalismo inmigrante y desarrollo. La experiencia de Estados Unidos y América Latina. Ed. FLACSO. México.

[10] Geertz, Clifford. (1982) La interpretacion de las culturas. Gedisa. España.

[11] Wellman, Barry, Peter J. Carrington, and Alan Hall (1988)."Networks as personal communities" en Wellman \& Berkowitz (eds.), Social Structures; A Network Approach. Cambridge University Press, Cambridge, 1988, pp. 130- 184.

Wellman, Barry (1996): "Are personal communities local? A Dumptarian reconsideration", Social Networks 18 (1996) 347-354.

[12] Molina, José Luis (2007). "El estudio de las redes personales: contribuciones, métodos y perspectivas", Empiria, Julio-Diciembre 10 (71106).

E-mail:javila.molero@gmail.com

javilamolero@hotmail.com

\footnotetext{
${ }^{i}$ En sus comienzos, esta perspectiva se enfocaba en el estudio de variables de composición entre los miembros de cada red personal (sexo, edad, ocupación, nivel estudios, lugar de residencia, etc.) y su relación con los vínculos de apoyo social. "Es probable que la diferencia responda a las distintas estrategias de integración desarrolladas por los grupos. En el caso argentino, al estar más integrado en la sociedad receptora, no habría necesidad de hacer grandes cambios en sus redes personales; mientras que en el resto de grupos, al estar menor integrados, si exista esta necesidad.
} 